\title{
A Novel Approach to Anatomical Structure Morphing for Intraoperative Visualization
}

\author{
Kumar Rajamani, Lutz Nolte, and Martin Styner \\ M.E. Müller Institute for Surgical Technology and Biomechanics, University of Bern, \\ Switzerland, \\ kumar.rajamani@MEMcenter.unibe.ch,
}

\begin{abstract}
In computer assisted surgery 3D models are now routinely used to plan and navigate a surgery. These models enhance the surgeon's capability to decrease the invasiveness of surgical procedures and increase their accuracy and safety. Models obtained from specifically acquired CT scans have the disadvantage that they induce high radiation dose to the patient. In this paper we propose a novel method to construct a patient-specific model that provides an appropriate intra-operative 3D visualization without the need for a pre or intra-operative imaging. The $3 \mathrm{D}$ model is reconstructed by fitting a statistical deformable model to minimal sparse 3D data consisting of digitized landmarks and surface points that are obtained intra-operatively. The statistical model is constructed using Principal Component Analysis from training objects. Our morphing method then computes a Mahalanobis distance weighted least square fit of the model by solving a linear equation system. The refined morphing scheme has better convergence behaviour because of the additional parameter that relaxes the Mahalanobis distance term as additional points are incorporated. We present leave-one-out experiments with model generated from proximal femors and hippocampi.
\end{abstract}

\section{Introduction}

Three dimensional (3D) models of the patient are routinely used to provide image guidance and enhanced visualization to a surgeon to assist in navigation and planning. These models are usually extracted from 3D imagery like CT or MRI. To avoid the high radiation dose and costs associated with such scans, image free approaches have been researched extensively and are becoming popular especially in orthopedic surgery. In an image free approach, building a 3D model that is specific to the patient anatomy is quite challenging as only very sparse patient data is available.

For this purpose, statistical models of shape have been extensively researched. The basic idea in model building is to establish from a training set the pattern of legal variations of shape. The model is adapted to the patient anatomy using digitized landmarks and bone surface points obtained during surgery. The main problem here is to extrapolate this extremely sparse three-dimensional set of points to obtain a complete surface representation. The extrapolation or morphing procedure is done via a statistical principal component analysis (PCA) based 
shape model. Fleute et al fit the morphed model surface to sparse intra-operative data via jointly optimizing morphing and pose [1]. Chan et al [5] optimize morphing and pose separately using an iterative closest point (ICP) method. In our prior work [6] we proposed to iteratively remove shape information coded by digitized points from the PCA model. The extrapolated surface is then computed as the most probable surface in the shape space given the data. Unlike earlier approaches, this approach was also able to include non-spatial data, such as patient height and weight. It is only applicable though for a small set of known points. In our earlier work [7] we presented a novel morphing scheme that computes a Mahalanobis distance weighted least square fit of the model by solving a linear equation system.

We propose a enhanced morphing scheme that has better convergence behaviour. This is achieved by having an additional parameter in the objective function that relaxes the Mahalanobis distance term as additional points are digitized. As more information in terms of additional digitized points is received we relax the constraint on the surface to remain close to the mean and allow it to deform so that the error between the predicted surface and the set of digitized points is minimized as far as possible. In this paper we demonstrate proof of principle of our method using a proximal femur model as well as hippocampus model and evaluate these models using leave-one-out experiments.

\section{Method}

\subsection{Model Construction}

The first step is to build a deformable model from a training database. The basic idea of building a statistical model based on PCA is to establish, from the training set, the pattern of legal variations in the shapes for a given class of images. Statistical PCA models were introduced by Cootes et al[2] based on point distribution model (PDM).

A key step in this model building involves establishing a dense correspondence between shape boundaries over a reasonably large set of training images. Our previous comparison study [4] of some of the popular correspondence establishing methods revealed that for modeling purposes the best of the correspondence method was Minimum Description Length (MDL) 3]. Correspondence was initialized with a semi-automatic landmark driven method and then optimized based on the MDL criterion.

We construct a deformable statistical shape model based on the corresponding point positions. Each member of the training population is described by individual vectors $\overline{\mathbf{x}}_{\mathbf{i}}$ containing all $3 \mathrm{D}$ point coordinates. The aim of building this model is to use several training datasets to compute the principal components of shape variation. PCA is used to describe the different modes of variations with a small number of parameters. For the computation of PCA, the mean vector $\overline{\mathbf{x}}$ and the covariance matrix $D$ are computed from the set of object vectors(1). The sorted eigenvalues $\lambda_{i}$ and corresponding eigenvectors $p_{i}$ of the covariance 


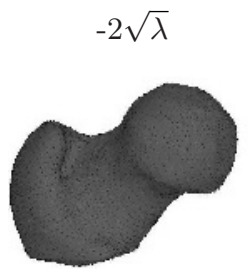

2

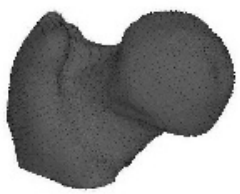

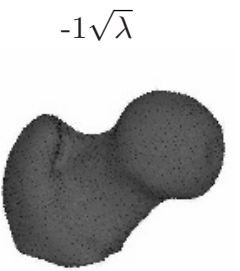

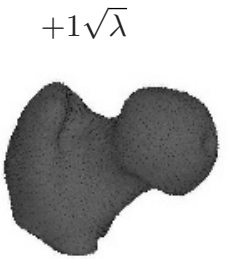

$+2 \sqrt{\lambda}$
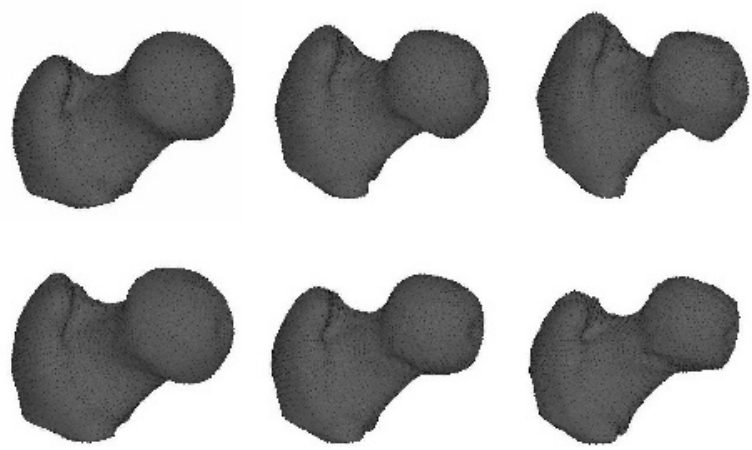

Fig. 1. The first two eigen modes of variation of our proximal femur model. The shape instances were generated by evaluating $\bar{x}+\omega \sqrt{\lambda_{k}} u_{k}$ with $\omega \in\{-2, . ., 2\}$

matrix are the principal directions spanning a shape space with $\bar{x}$ representing its origin(2). Objects $\mathbf{x}_{\mathbf{i}}$ in that shape space can be described as linear combination with weights $\mathbf{b}_{\mathbf{i}}$ calculated by projecting the difference vectors $\mathbf{x}_{\mathbf{i}}-\overline{\mathbf{x}}$ into the eigenspace(3).

$$
\begin{aligned}
D & =\frac{1}{n-1} \sum_{1}^{n}\left(\mathbf{x}_{\mathbf{i}}-\overline{\mathbf{x}}\right) \cdot\left(\mathbf{x}_{\mathbf{i}}-\overline{\mathbf{x}}\right)^{T} \\
P & =\left\{\mathbf{p}_{\mathbf{i}}\right\} ; D \cdot \mathbf{p}_{\mathbf{i}}=\lambda_{i} \cdot \mathbf{p}_{\mathbf{i}} ; \\
\mathbf{b}_{\mathbf{i}} & =D^{T}\left(\mathbf{x}_{\mathbf{i}}-\overline{\mathbf{x}}\right) ; \mathbf{x}_{\mathbf{i}}=\overline{\mathbf{x}}+P \cdot \mathbf{b}_{\mathbf{i}}
\end{aligned}
$$

Figure 1 shows the variability captured by the first two modes of variation of our proximal femur model varied by \pm 2 standard deviation.

\subsection{Morphing}

Anatomical structure Morphing is the process of recovering the patient specific $3 \mathrm{D}$ shape of the anatomy from the few available digitized landmarks and surface points. Our approach uses the statistical based shape model built earlier to infer the anatomical information in a robust way. This is achieved by minimizing the residual errors between the reconstructed model and the cloud of random points, and provides the best statistical shape that corresponds to the patient.

Earlier morphing methods were based on fitting procedures in Euclidean space and have the disadvantage that these are often computationally expensive and only a small set of shape variations can be considered. The morphed model also does not represent the most probable shape given the input data but rather a constrained fit. Our novel morphing method operates directly in the PCA shape space incorporating the full set of possible variations. The method consists of two steps 
- Initially a small point-set of anatomical landmarks with known correspondence to the model is digitized. This is used to register the patient anatomy to the model. This also provides an initial estimation of the $3 \mathrm{D}$ shape with only a few digitized points.

- To improve the prediction additional points can be interactively incorporated via closest distance correspondence. A color coded feedback is given to the surgeon which shows regions where the prediction is accurate and regions where the prediction could be improved. This assists the surgeon in deciding the location where to digitize extra points.

The morphing computation is based on formulating the problem as a linear equation system and then solving for the shape parameters that best describe the unknown shape. An additional term in the objective function minimizes the Mahalanobis shape distance. The objective function that we minimize is defined as follows

$$
f=\rho *\left\{\gamma * \sum_{\substack{k=1 \\ j=\operatorname{index}_{k}}}^{N}\left\|\boldsymbol{Y}_{\boldsymbol{k}}-\left(\boldsymbol{X}_{\boldsymbol{j}}+\sum_{i=1}^{m} \alpha_{i} \boldsymbol{p}_{\boldsymbol{i}}(j)\right)\right\|^{2}\right\}+(1-\rho)\left\{\sum_{i=1}^{m} \frac{\alpha_{i}^{2}}{\lambda_{i}}\right\}
$$

with $N$ the number of points that are digitized, $\boldsymbol{Y}_{\boldsymbol{k}}$ is the kth digitized point, $\boldsymbol{X}_{\boldsymbol{j}}$ is the point in the mean model that is closest to $\boldsymbol{Y}_{\boldsymbol{k}}, \boldsymbol{p}_{\boldsymbol{i}}(j)$ is the $j_{\text {th }}$ tuple of the $i_{t h}$ shape basis vector, $\lambda_{i}$ the $i_{t h}$ eigen value and $\alpha_{i}^{\prime} s$ are the $m$ shape parameters that describe the shape. The first term of the function minimizes the distance between the predicted shape and the set of digitized points. This is similar to the Euclidean distance term used by Fleute [1]. The second term controls the probability of the predicted shape. This term ensures that the predicted shape has minimal Mahalanobis shape distance. The factor $\gamma$ is a parameter that weights the two terms of the function and ensures that a valid shape is predicted in the scenario when there are relatively few digitized points. A series of tests with varying values of gamma was carried out to determine the optimal value of gamma. The granularity of gamma was chosen using binary selection scheme where the region containing the current best value of gamma was further divided to find gamma to an acceptable level of accuracy. Our series of tests revealed that for our current application the best results with the least prediction mean and median errors were obtained when the value of gamma was fixed at one. Hence based on our tests the optimal value of $\gamma$ was empirically fixed at one.

We modified the morphing scheme to one that is enhanced and has better convergence behaviour. This is achieved by having an additional parameter $\rho$ in the objective function that relaxes the Mahalanobis distance term as additional points are digitized. As more information in terms of additional digitized points is received we relax the constraint on the surface to remain close to the mean and allow it to deform so that the error between the predicted surface and the set 

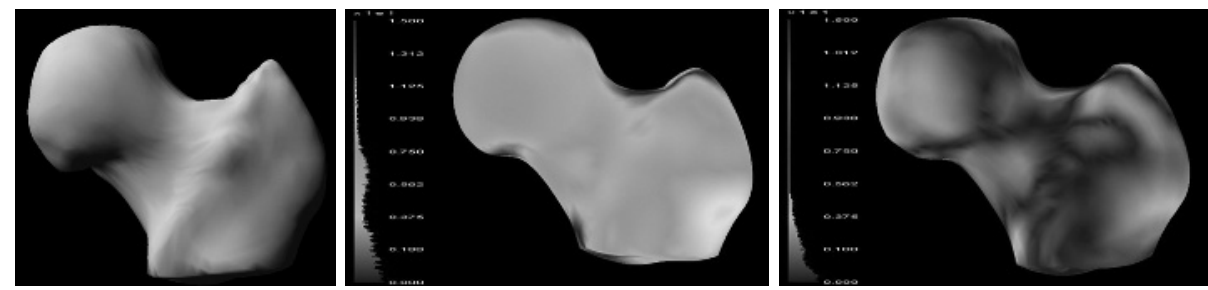

Fig. 2. Left: A typical proximal femur of the population that was used in the leaveone-out test. Middle: The average shape of the population with color coded distance map to the actual shape. The mean error is $3.37 \mathrm{~mm}$ and the median is $2.65 \mathrm{~mm}$. Right:The shape based on only 6 digitized points with color coded distance map to the actual shape. The mean error is $1.50 \mathrm{~mm}$ and the median error is $1.25 \mathrm{~mm}$

of digitized points is minimized as far as possible. As the error ideally decreases exponentially with the increase in the number of digitized points, we chose $\rho$ to increase logarithmically, and was defined according to the following equation

$$
\rho= \begin{cases}0.5 & N \leq 6 \\ \frac{\log \left\{\frac{N}{M a x N}(g * e-1)+1\right\}}{2 * \log (g * e)}+0.5 & N>6\end{cases}
$$

where $\mathrm{N}$ is the number of digitized points, MaxN is the total number of points $\mathrm{g}$ is a factor which determines the rate of growth of $\rho$. To achieve faster growth rate for $\rho$, g was empirically set to be the number of members in the population.

To determine the shape parameters $\alpha_{i}$ that best describe the unknown shape, the function $f$ is differentiated with respect to the shape parameters and equated to zero. This results in a linear system of $m$ unknowns, which is solved with standard linear equations system solvers using QR decomposition.

\section{Results}

In this paper we demonstrate proof of principle of our method using the proximal femur structure. $14 \mathrm{CT}$ scans of the proximal femur were segmented and a sequence of correspondence establishing methods was employed to compute the optimal PCA model [4]. A series of leave-one-out experiments was carried out to evaluate the new method. Three anatomical landmarks, the femoral notch and the upper and the lower trochanter are used as the first set of digitized points. This is used to initially register the model to the patient anatomy. The remaining points are added uniformly across the spherical parameterization so that they occupy different locations on the bone surface.

Our studies with the two different correspondence methods, MDL and closest correspondence for incorporating additional points along with different error plots are discussed in [7]. Figure 2 shows a example of a very good estimate with mean error of $1.5 \mathrm{~mm}$ obtained with as few as 6 digitized points using MDL 

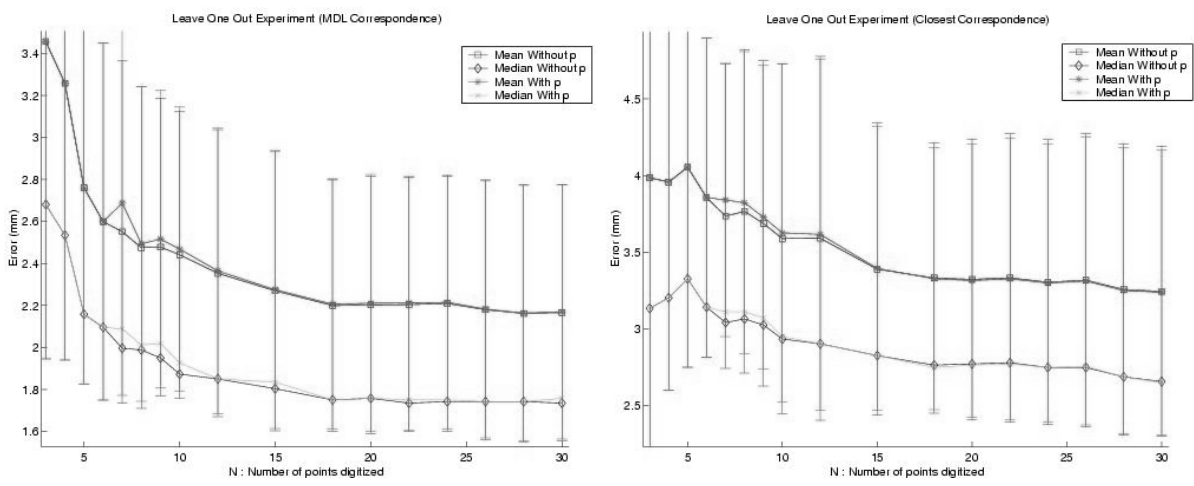

Fig. 3. Statistics cumulated from the different leave-one-out experiments of the proximal femur with and without the $\rho$ factor. The average of the mean error and the average of the median is plotted against the number of digitized points Left: Shows the error plot obtained using MDL correspondence. Right: Shows the error plot using Closest Point Correspondence

correspondence. The color-coded 3D rendering is calculated using Hausdorff's Distance to measure the distance between discrete 3D surfaces 8 .

Here we present results using our refined morphing scheme and also compare it to our initial version. Figure 3 shows the cumulative statistics of all leave-oneout experiments with and without $\rho$ factor using the MDL and Closest point correspondence. In both the cases there seemed to be no significant improvement using the $\rho$ factor, mainly due to low number of subjects in our proximal femur study population.

To evaluate the influence of the $\rho$ factor we studied the enhanced morphing scheme in Hippocampus model generated from 172 hippocampus instances 9 . Here the larger population helps us to efficiently capture the shape variability and also helps us to evaluate better the influence of the $\rho$ factor. Figure 4 shows the cumulative statistics from ten randomly chosen leave-one-out experiments with and without $\rho$ factor using the MDL and Closest point correspondence for the Hippocampus population. Here we can clearly see the excellent influence of the $\rho$ factor. The better convergence and the error factor We gain is about $10 \%$ in the MDL scenario and about $5 \%$ in the closest correspondence case.

\section{Discussion}

In this paper we have presented a refined novel anatomical structure morphing technique to predict the three dimensional model of a given anatomy using statistical shape models. Our scheme is novel in that it operates directly in the PCA shape space and incorporates the full set of possible variations. It is also fully interactive, as additional bone surface points can be incorporated in real-time. The computation time is mainly independent of the number of points 

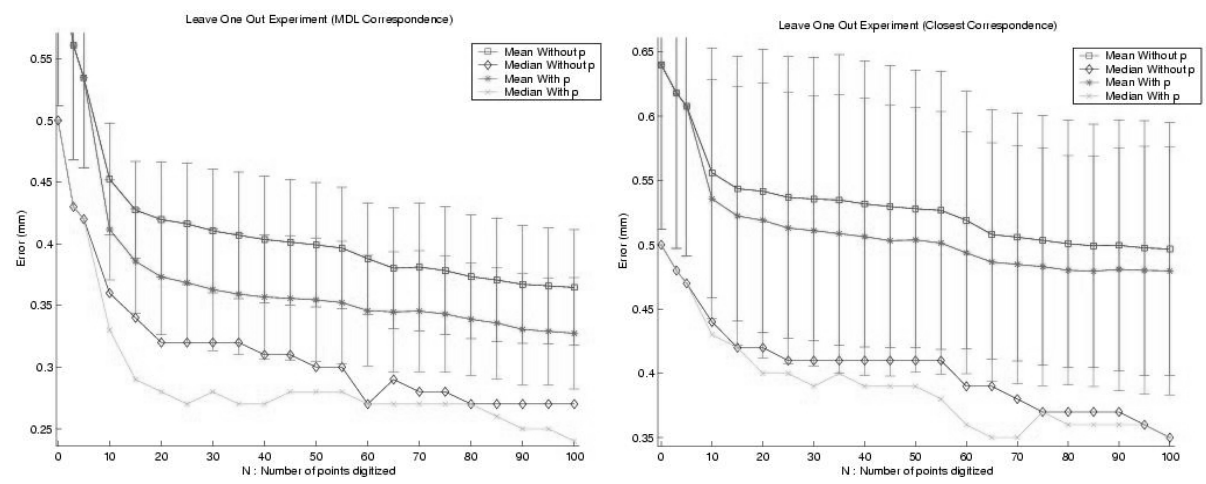

Fig. 4. Statistics cumulated from ten randomly chosen hippocampus leave-one-out experiments with and without the $\rho$ factor. The average of the mean error and the average of the median is plotted against the number of digitized point. Left: Shows the error plot obtained using MDL correspondence. Right: Shows the error plot using Closest Point Correspondence

intra-operatively digitized, and largely depends on the number of members in the population. The enhancement of this scheme compared to our earlier approach is that we achieve smaller errors and better convergence as additional points are digitized.

The gamma parameter plays a vital role in balancing the predictive error term and the probability term. We empirically fixed its value to adapt to the case when small number of points are digitized. The $\rho$ parameter helps us to relax the probability term to get a much better estimate as more points are digitized. The effect of the $\rho$ parameter is not significantly noticed in the case when the population size is small. This is because the error gets stabilized and uniform after the first few points are digitized and there is not much information that could be extracted by adding additional points in this case. Hence the $\rho$ factor seem not to contribute much as was observed in the proximal femur model with a population size of only 14 members. On the contrary in the hippocampus population the effect of the $\rho$ parameter was significantly visible and it contributes in a significant way to decrease the error and achieve better convergence.

Another interesting observation that we can make is that the average mean error in the hippocampus population is far less compared to the proximal femur population. With 20 digitized points the average mean error in the proximal femoral population is about $2.25 \mathrm{~mm}$ whereas in the hippocampus population it is only $0.37 \mathrm{~mm}$. The reason for this is because the hippocampus is a simple shape and we had a large population for the hippocampus model. Interestingly the error reduction that we achieve with 20 digitized points is about $35 \%$ for both the models.

There are a number of extensions that we plan to incorporate to this idea. We have a fully developed and validated technology at M.E. Müller Institute to 
extract bone contours from Ultrasound (US) images. First we plan to this use this large set of bone surface points from US images into the morphing scheme. Using this technique we can non-invasively get a large set of bone surface points intraoperatively. We also plan to incorporate fluoroscopic images into the process to extract surface points.

The concept of anatomical structure morphing has many interesting medical applications. The primary application that we focus is on hip surgery such as total hip replacement (THR) and knee surgery such as total knee arthroplasty (TKA) and anterior cruciate ligament surgery (ACL). Several current navigation systems for TKA/THR do not require preoperative CT or planning. By moving the joint, the center of motion is obtained. The hip, knee, and ankle motion centers give the functional axes of the femur and tibia. The surgeon is usually provided with a digital readout and a single display of the relative bone positions or angles. It is sometimes difficult for surgeons to intuitively understand such displays. The technique of anatomical structure morphing introduces novel navigation concepts wherein reconstructed 3D bony images are overlaid on top of $2 \mathrm{D}$ views of the axes. The proposed technology brings a variety of advantages to orthopaedic procedures, such as improved accuracy and safety, often reduced radiation exposure, and improved surgical reality through $3 \mathrm{D}$ visualization and image overlay techniques. In particular navigation based on anatomical structure morphing opens the door to larger minimally invasive approaches.

\section{References}

1. Fleute, M., Lavallee, S.: Building a Complete Surface Model from Sparse Data Using Statistical Shape Models, MICCAI (1998) 879-887

2. Cootes, T., Hill, A., Taylor, C.J., Haslam, J.: The Use of Active Shape Models for Locating Structures in Medical Images. Img. Vis. Comp. (1994) 355-366

3. Davies, Rh.H, Twining, C.J., Cootes, T.F., Waterton, J. C., Taylor, C.J.: A Minimum Description Length Approach to Statistical Shape Model. IEEE TMI (2002)

4. Styner, M.A., Kumar .T.R., Nolte L.P., Zsemlye G., Szekely, G., Taylor, C.J., Davies Rh.H.,: Evaluation of 3D Correspondence Methods for Model Building, IPMI (2003) 63-75

5. Chan, C.S., Edwards, P.J., Hawkes, D.J., : Integration of ultrasound-based registration with statistical shape models for computer-assisted orthopaedic surgery, SPIE, Medical Imaging (2003) 414-424

6. Kumar T.R., Nolte L.P., Styner M.A.,: Bone morphing with statistical shape models for enhanced visualization, SPIE Medical Imaging (2004)

7. Kumar T.R., Joshi, S.C., Styner M.A., : Bone model morphing for enhanced surgical visualization, IEEE International Symposium on Biomedical Imaging: From Nano to Macro ISBI (2004)

8. Aspert,N., Santa-Cruz, D., Ebrahimi, T.,: MESH:-Measuring Errors between Surfaces using Hausdorff Distance, IEEE ICME (2002) 705-708

9. Styner, M.A., Lieberman, J., Gerig, G. Boundary and Medial Shape Analysis of the Hippocampus in Schizophrenia, MICCAI (2003) 